\title{
NOUVELLES DONNÉES SUR LA PSYCHOPHYSIOLOGIE DE L'ÉLEVAGE DES REINES CHEZ APIS MELLIFICA
}

\author{
(DEUXIËME NOTE)
}

\author{
PAR
}

\section{VUILLA UME}

Laboratoire de Zoologie (S. P. C. N.), Faculté des Sciences de Rennes et Station de Recherches apicoles, Bures-sur-Yrette.

Dans une note précédente Vuiliavme (I957) nous arons étudié l'action de différents facteurs sur les élevages de reines par les Abeilles. Nous avons mis en évidence les faits suivants :

Io Les variations du pourcentage d'acceptation des cupules royales artificielles tiendraient à un équilibre entre deux substances incluses dans la cire: l'une inhibitrice de l'acceptation: 1'autre facilitatrice, secrétée peut-ètre par les ouvrières et beaucoup moins stable que la première.

$2^{\circ}$ Parmi les stimuli qui poussent les nourrices à accepter une jeune larve qui leur est présentée dans une cupule de cire, comptent : la forme du fond, la forme des bords, la position des cupules, leur écartement. Mais, la matière dont est construite la cupule parait de peu d'importance puisque les ouvrières acceptent la paraffine, toutes sortes de cires minérales ou végétales et même le verre ou certaines matières plastiques.

$3^{\circ}$ Pour que la jeune larve introduite dans une cupule artificielle fasse l'objet d'un élevage royal, elle doit être vivante et appartenir au genre Apis; son âge doit être inférieur à 3 jours; le sexe n'est pas un facteur très important et des larves mâles peuvent être acceptées.

$4^{0}$ I,es jeunes larves étrangères à la colonie éleveuse sont acceptées sans difficulté; la présence de la reine ne suffit pas à inhiber l'élevage royal, pourvu qu'il ait débuté dans une colonie orpheline (ou ruchette d'acceptation).

Dans une note technique sur l'élevage des reines Vurlatur (r957), nous traitions particulièrement du rôle du " paquet " d'Abeilles chargé de 
démarrer l'élevage royal. Au cours d'un passage de 24 heures dans cette colonie orpheline ou "starter ", la familiarisation des cellules garnies de larves s'accentue et le dépôt de gelée royale est amorcé. Nous avons pu alors supprimer le passage dans le starter en "faniliarisant " les cupules avant leur emploi et en déposant les larves sur de la gelée royale pure ou sur un mélange concentré de gelée royale (un volume) et d'eau (un volume) : I a familiarisation proprement dite se fait au cours d'un passage préalable, des cupules neuves et vides, pendant 24 heures, dans une ruche quelconque.

\section{I. - NOUVEAUX DÉTAILS SUR L'ACTION DE LA GELÉE ROYALE.}

Nous avons précisé le rôle de la gelée royale dans l'amorçage, déjà étudié dans notre mémoire de I957. Les tableaux suivants (I, II et III), montrent les résultats obtenus au cours des essais qui ont permis de supprimer les starters. Dans toutes les expériences les cellules sont mises dans la même ruche en compétition, en position alterne sur les baguettes porte-cellules de façon à éviter le facteur de position qui peut jouer un rôle important.

TABLEAU I

\begin{tabular}{|c|c|c|}
\hline & Larves déposées sur de l'eau pure & $\begin{array}{l}\text { Larves déposées sur un mélange } \\
\text { d'eau }(\text { r } 00 \mathrm{~g})+\text { gelée royale }(3 \mathrm{~g})\end{array}$ \\
\hline I & $52 \%$ & 68,7 \\
\hline 2 & $32,2 \%$ & 36,9 \\
\hline .3 & $f_{2}$ & $5,3,7$ \\
\hline 4 & 12,8 & $\mathrm{I} 1,9$ \\
\hline 5 & 6,6 & 8,2 \\
\hline 6 & 4,1 & 4,4 \\
\hline
\end{tabular}

Légende...- I : Pourcentage de cellules actejtées par rapport au nb. de cellules greffées. — 2 : Pourcentage de cellules teminćes par rapport au nb de cellules greffées. - -3: Pourcentare de cellules terminécs jar rapport au nb de cellules accejtées. - 4: Nombre de cellules greffées pour récolter un gramne de gelée royale. - $5:$ Nonbre de cellules accep tées pour récolter un gramme de gelée royale. - $6:$ Nonbre de cellules terminées pour I gramme de gelée royale.

Les chiffres de ce tableau montrent un léger avantage en faveur des cellules amorcées avec le mélange eau + gelée royale. Eilles sont un peu mieux acceptées et il suffit de II,9 cellules greffées pour récolter I g de gelée royale alors qu'il en faut I2,8 dans le cas des larves déposées sur de l'eau pure.

Sur le tableau suivant figurent les résultats obtenus avec les cellules amorcées à la gelée royale pure.

L'action de la G. R. au moment de l'introduction dans la ruche joue un rôle très important sur la quantité de $G$. R. dégorgée par les nourrices. 
Comme nous l'avions déjà signalé, si le pourcentage des cellules acceptées n'est guère modifié, le pourcentage des cellules terminées est supérieur, et les cellules terminées renferment davantage de $G$. R., que dans le cas de l'eau pure.

Tableau II

\begin{tabular}{|c|c|c|}
\hline & Larves déposées sur eau pure & $\begin{array}{l}\text { Larves déposées sur gelée rovale pure } \\
(0,033 \mathrm{~g} \mathrm{p} / \text { cellule })\end{array}$ \\
\hline $\begin{array}{l}\mathrm{I} \\
2 \\
3 \\
4 \\
5 \\
6\end{array}$ & $\begin{array}{l}43,2 \% \\
20,4 \% \\
56,7 \% \\
16 \\
7 \\
+\end{array}$ & $\begin{array}{r}45,1 \\
30,8 \\
68,3 \\
10,3 \\
4.6 \\
3,2\end{array}$ \\
\hline \multicolumn{3}{|c|}{ (Même légende que pour le tableau I) } \\
\hline
\end{tabular}

Nous avons comparé ensuite l'amorçage à la gelée royale pure en compétition avec un mélange eau + gelée royale (Résultats sur le tableau suivant).

\section{TABLEAU III}

\begin{tabular}{c|c|c}
\hline & Larves déposées s/G. R. pure & $\begin{array}{c}\text { Larves déposées s/mélange } \\
\text { eau (I00 g) }+ \text { G. R. (3 g) }\end{array}$ \\
\hline 1 & 53,8 & $5 \mathrm{~T}, 7$ \\
2 & 34,2 & 30,6 \\
3 & 63 & 59,1 \\
4 & 12,5 & 1,7 \\
5 & 6,7 & 7,6 \\
6 & 4,2 & 4,5 \\
& (Même légende que pour le tableau I) \\
\hline
\end{tabular}

Si les quantités de cellules acceptées et terminées sont sensiblement les mêmes quand on amorce à la gelée royale pure ou à l'aide d'un mélange dilué (eau + G. R.), il faut cependant moins de cellules greffées pour récolter I $g$ de $G$. R. pure et les cellules terminées renferment dans ce même cas, davantage de G. R.

\section{TABLEAU IV}

\begin{tabular}{c|c|c}
\hline \hline & $\begin{array}{c}\text { Larves déposées sur beaucoup de } \\
\text { G. K. (O,I g) }\end{array}$ & $\begin{array}{c}\text { Larves déposées sur peu de G. R. } \\
(0,033 \mathrm{~g})\end{array}$ \\
\hline 1 & $75 \%$ & 75 \\
2 & $32 \%$ & 9 \\
3 & $24 \%$ & 9 \\
& (Même légende que pour le tableau I) \\
\hline
\end{tabular}

La quantité de G. R. déposée dans les cupules au moment de leur amorçage joue un rôle important dans le pourcentage des cellules termi- 
nées (9 p. Ioo avec peu de gelée royale pure au lieu de 24 p. Ioo avec plus de gelée).

En introduisant directement dans le finisseur, sans passage au starter, des cellules renfermant une jeune larve déposée sur de la $\mathrm{G}$. R. ou sur Eau + G. R. nous retrouvons des résultats confirmant les précédents.

Avec des cellules neuves, de cire, non familiarisées, nous comptons I8,7 p. Ioo de cellules terminées dans le cas de la G. R. pure et o p. Ioo dans le cas du mélange dilué : eau + G. R. Avec des cellules familiarisées nous avons obtenu les résultats suivants :

$5^{6} \mathrm{p}$. Ioo de cellules terminées par rapport aux cellules greffées pour les larves déposées sur de la gelée royale pure, contre 6,2 p. Ioo avec le mélange Eau + G. R.

Pour 80 cellules récupérées (donc familiarisées) testées en compétition (G. R. pure contre mélange eau + G. R.) nous avons récolté $\mathrm{I} 7,4 \mathrm{~g}$ de G. R. dans le premier cas pour 7,2 g dans le second.

Ceci nous montre l'importance de l'amorçage à la G. R. concentrée sur les rendements des élevages royaux.

\section{Remarque.}

Cet effet important de la G. R. se conserve longtemps comme le montre le tableau VI. Nous avons, pour étudier cette conservation, utilisé de la G. R. fraîche (le jour de sa récolte) et de la gelée royale conservée au frigidaire à $0-4^{\circ}$ pendant un an. Si la gelée royale "vieillit ", tout au moins en dehors de la ruche (Chauvin et GoILlo's) comme le montre l'étude des variations de ses propriétés physiques et physiologiques, elle demeure malgré tout fort longtemps un aliment convenable pour les jeunes larves (noter ici que d'après WEAVER, les élevages in iitro pour donner des Reines parfaites, nécessitent par contre de la G. R. ultrafraîche). Nous ne voulons parler ici que de l'élevage dans ses premiers moments, et n'envisageons pas le degré final de développement des organes. Mais dans ces premières phases, les substances de la G. R. qui favorisent la réussite paraissent stables, s'il n'en est pas de même pour les substances de la G. R. qui induisent le développement parfait et complet des ovaires de la Reine.

TABLEAU VI

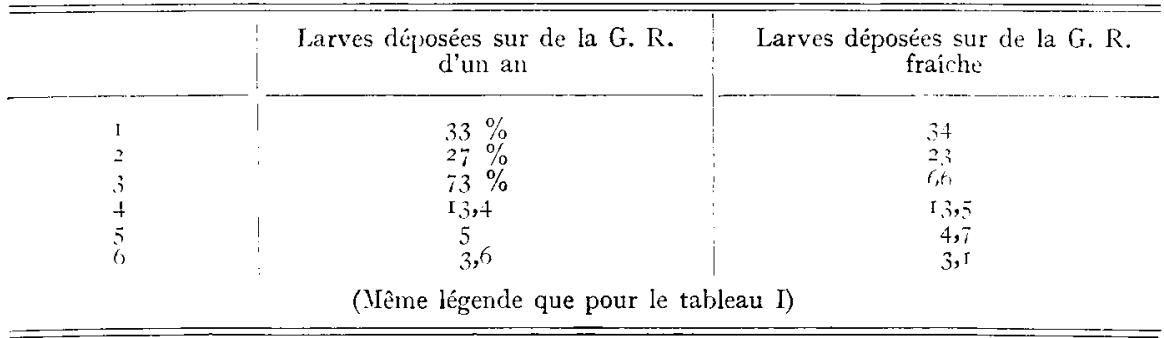




\section{Substitutions répétées de larves en élevage et quantité de G. R. dégorgée}

Le rôle de la $G$. R. est mis en évidence également dans l'expérience suivante :

Si dans les cellules déjà très avancées, nous substituons à des larves royales âgées de 3 jours des jeunes larves en laissant toute la G. R. à l'intérieur des cellules et en introduisant à nouveau les cellules royales directement dans une ruche finisseuse, les pourcentages des larves élevées sont très importants :

Au cours de la première opération, $72 \mathrm{p}$. Ioo des jeunes larves sont élevées tandis que la réserve de G. R. mise à la disposition des larves augmente. Une seconde substitution des larves dans les mêmes cellules au cours $\mathrm{du} 3^{\mathrm{e}}$ jour de vie larvaire réussit encore et $7^{8} \mathrm{p}$. Ioo des cellules sont acceptées : la quantité de G. R. continue à augmenter. A la suite d'une $3^{\mathbf{e}}$ substitution, $77 \mathrm{p}$. roo des larves sont encore élevées, mais sans apport nouveau de G. R. La réserve de G. R. se dessèche et change totalement d'aspect. Garde-t-elle ses propriétés? C'est un point que nous n'avons pas élucidé, et qui nous paraît peu probable.

Au cours de ces substitutions nous sectionnions les cellules royales un peu au-dessus du niveau de la $G$. R. qu'elles contenaient, ceci afin d'extirper plus facilement les larves. Il ne nous a pas semblé que cette pratique perturbe sérieusement 1'élevage. WEAVER (I957) remarque pourtant que la section du " chapeau " de cire quand la gelée royale atteint le bord d'une cupule de verre amène l'expulsion de la larve et l'abandon de l'élevage.

Ces cellules royales dans lesquelles plusieurs larves sont élevées successivement atteignent, quand elles sont terminées, et ceci déjà après 1a première substitution de larve, une taille bien supérieure à celle des cellules royales normales. Il est à peine besoin de souligner l'application pratique possible de ces expériences pour l'obtention de reines plus grosses et sans doute à fécondité plus grande. WEAVER remarque que les reines issues de ce double greffage, qui ont donc reçu abondance de G. R. ont seulement la spermathèque plus développée, mais pas plus d'ovarioles. Mais ses expériences sont trop peu nombreuses pour conclure. Le double greffage est connu depuis longtemps des éleveurs américains, mais il ne semble pas avoir fait l'objet d'études suffisamment approfondies.

Signalons que si la gelée royale semble, en même temps que la larve, déterminer l'élongation des cellules géantes, un autre facteur intervient peut-être en même temps : c'est celui de la substance d'acceptation qui doit être de plus en plus abondante au fur et à mesure que l'élevage royal se prolonge. 
Influence de la larie.

Si la larve est indispensable à l'édification du chapeau de la cellule royale, elle n'est pas toutefois un facteur suffisant, de même qu'elle ne semble jouer aucun rôle dans l'édification de l'ébauche de la cellule royale (DARChen et Vuiflaume ).Dans le cas de tous les élevages naturels en effet, les ébauches de cellules royales sont construites d'abord, et la reine ne vient y pondre qu'ensuite. Tant que la larve n'est pas née, l'ébauche de la cellule royale reste à ce stade. Ce n'est qu'à la naissance de la larve que le dépôt de gelée royale commence en même temps que s'édifie la cellule royale typique. Ces deux phénomènes : dépôt de $G$. $R$. dans les cellules et allongement de l'ébauche royale vont toujours de pair. L'un ne s'effectue jamais sans l'autre. Il semble que Detreurance observe le même phénomène chez les Polistes : la construction du pédicelle d'une part, celle de la base des cellules d'autre part, celle des cellules enfin(allongement à partir de l'ébauche) dépendent de facteurs divers; mais l'élongation de la cellule ne se fait chez les Polistes qu'en présence de larves saines; les œufs ou le couvain abortif ne déclenchent pas ce comportement spécial.

Chez les Abeilles, la fourniture des cupules par l'expérimentateur correspond peut-être à un télescopage des "façons pédicellaires " et des "façons CC" (base des cellules )observées par Deleurance. L'édification du chapeau correspondrait aux "façons CI " (élongation des cellules garnies d'une larve saine). Le dépôt de G. R. à l'intérieur d'une cellule où se trouve une jeune larve ou une pré-reine constituerait alors un autre phénomène, propre aux Abeilles.

\section{II. - QUE NOUS APPREND LA PRÉSENTATION D'ÉBAUCHES DE FORMES TRĖS ANORMALES ?}

Poursuivant nos expériences sur les cupules de formes diverses, nous avons déposé de jeunes larves sur des cupules de forme très particulière :

I $^{0}$ Dans des cupules de forme semblable aux normales, mais de 2 centimètres de diamètre au lieu de 8 millimètres, sans cloisons au centre; nous y placions I, 2, 3 ou 4 larves sur une goutte de G. R. pure. Mais ces expériences, que les cupules soient neuves ou familiarisées, n'ont donné aucun résultat. Jamais autour des larves nous n'avons trouvé de constructions cirières amorçant la construction d'une cellule royale. Les Abeilles construisaient autour, en se servant de la cupule comme d'un point de départ, des cylindres de cire alvéolée; leur intérieur et leur extérieur étaient constitués par des cellules d'ouvrières normales, avec toutefois les variations de forme qu'impose la courbure de l'ébauche de cire. DARchex a obtenu 
des constructions analogues en présentant aux abeilles un manchon de cire gaufrée (photos 6, 7,8).

Quel est le facteur qui contrarie ici l'élevage royal? Simplement sans doute la forme de la partie basale de la cellule royale, dont la construction dépend d'autres facteurs que le reste de la cellule, comme je l'ai déjà montré. Cette partie basale correspond aux "façons $C_{1}$ " des Polistes de Deleurance; ; et de même que les Polistes engagés dans les "façons pédicellaires Cpd " ne peuvent " dévier " du côté des "façons $\mathrm{CC}$ ou $\mathrm{C}_{1}$ ", de même "façons basales " et "façons du chapeau " ne sont pas interchangeables chez les Abeilles. L'ouvrière peut continuer une cellule dont la base (forme interne) lui convient, mais elle ne peut rectifier cette base si elle ne lui convient pas. Les résultats médiocres obtenus précédemment avec des cupules cubiques (Vuilfaume I957) dont le fond n'est lui non plus jamais modifié, nous confirment la dissociation des deux processus, construction de la base et construction du chapeau.

$2^{\circ}$ Nous avons alors essayé de faciliter la construction en plaçant des ébauches de cloisons de cire entre les larves avant le dépôt de celles-ci dans les grandes cupules précédemment employées; comme dansle cas précédent, l'ensemble passait dans une ruche avant son emploi, pour la familiarisation. Mais dans ce cas encore nous n'obtenons jamais d'élevage royal. Pourquoi les Abeilles abandonnent-elles les larves côte à côte ou séparées par des cloisons? Pourquoi ne les élèvent-elles pas tout au moins en ouvrières? C'est une question que nous nous sommes déjà posé maintes fois. Nous sommes loin ici du comportement des Bombus, hyménoptères également sociaux, mais plus primitifs : ici toutes les larves reposent côte à côte, sans cloisons entre elles, sur la bouillie nutritive, dans de grands sacs de cire de forme irrégulière.

$3^{\circ}$ Devant cet échec, nous nous sommes souvenus que nous avions obtenu précédemment des résultats satisfaisants en plaçant des cupules normales côte à côte sur une réglette; parfois plusieurs cellules royales (jusqu'à 6), parfaitement normales, étaient collées les unes aux autres. Nous avons alors construit des sortes de barquettes de cire de $8 \mathrm{~mm}$ de large et io cm de long, profondes de io mm. Des larves placées sur de la G. R. à l'intérieur de ces cupules spéciales, sans cloisons entre elles, sont égalennent toutes abandonnées. Là encore, la zone critique ou partie basale resterait à modifier et ne peut l'être. D'ailleurs en plaçant, ici encore, entre les larves des cloisons de cire, les résultats restent tout aussi mauvais. Pourtant les cellules artificielles comprises entre les cloisons dans les barquettes étaient assez semblables cette fois aux cellules cubiques à fond arrondi utilisées dans un travail précédent (VuIlLAUme r957); et si ces cellules étaient abandonnées dans une grande proportion, les Abeilles y élevaient néanmoins quelques larves. De plus, la structure des cellules abandonnées n'était pas modifiée, alors que nous ne retrouvons rien ici 
de la structure initiale des barquettes. Après 24 heures, les loges initiales sont divisées en 2 par les Abeilles et chaque cellule finale est arrondie (photos $n^{0} 3,4,5$ ). Rappelons ici 1'hypothèse que nous formions ailleurs au sujet de la forme des cellules, en supposant que la forme arrondie était la plus primitive, vers laquelle les abeilles tendent à revenir dans de nombreux cas; toutes les cellules de reine, qui sont toujours plus ou moins isolées, affectent précisément cette forme; il en est de même des cellules de mâles isolées, dans certains cas (mâles élevés dans des cellules de reine) ; enfin, quand les Abeilles transforment la structure d'un rayon (passage de cellules de mâles à cellules d'ouvrières par exemple) elles arrondissent également toutes les ouvertures (DARCHEN et VUILLAUME). Ici, les cellules construites à partir des barquettes ou des larges cupules cloisonnées sont également toutes rondes (photo 4). Les Abeilles construisent également des cellules rondes, quand on n'a pas placé les cloisons, mais dans un ordre quelconque et leurs dimensions sont alors assez variables. D'après DELEURANCE, quand une femelle fondatrice de Poliste édifie une première cellule isolée, sa base est toujours cylindrique; elle ne devient prismatique que lorsque d'autres cellules sont construites à son contact; mais chez nos Abeilles, la forme arrondie se maintient dans certains cas, même lorsque les cellules sont en contact étroit. Nous avons signalé aussi que dans les cupules cubiques acceptées, le chapeau est toujours cylindrique, bien que la base non remaniée reste cubique.

$4^{\circ}$ Dans le cas de cupules en matière plastique trop larges où l'on greffe une larve, nous avons vu les Abeilles réduire le diamètre des moules (Vuiliaume, I957) et construire, en utilisant seulement une partie des parois de plastique, une cellule royale de cire comprise entièrement à l'intérieur de la cupule. Pourquoi alors, dans le cas de nos cupules de cire trop larges, ne construisent-elles pas aussi à leur intérieur, des cellules royales complètes et appuyées contre les parois? C'est que, malgré la grande largeur des cupules plastiques, leur base est de dimensions semi-nomales ( $9 \mathrm{~mm}$ contre $8 \mathrm{~mm}$ de diamètre pour les normales). Les Abeilles n'ont donc pas à reconstruire la zone critique et peuvent ramener le reste à des dimensions habituelles.

\section{Influence de la proximité des cellules; le rôle du bord libre.}

Nous avions étudié, dans une note précédente, 1'espacement optimum qu'il convient de donner aux cupules pour obtenir le maximum de réussites; nous avions vu qu'il est possible de placer des cupules normales et familiarisées côte à côte, et l'on obtient encore I7 p. Ioo de cellules terminées : mais la zone apicale est toujours libre, et son bord n'est soudé en aucun point aux cellules voisines (photos I, 2). Voilà sans doute la raison de 1'insuccès des barquettes de cire : la trop grande proximité des cases 
à cloisons mitoyennes rend impossible la séparation des bords. Si bien que nous devons ajouter à la liste des facteurs importants et non modifiables de la cellule royale, en plus de la forme du fond, les caractères du bord.

Mais ce comportement pose d'autres problèmes. D'abord, qu'est-ce qui pousse les Abeilles à expulser les lavves avant de transformer les barquettes de cire en cellules d'ouvrières? Remarquons alors que dans tous les cas où des cellules d'ouvrières sont transformées en cellules de mâle et vice-versa, et dans ceux où un fond est remis à des cellules dont on l'avait enlevé (DARCHEN) ni ceufs ni larves ne se trouvent dans les cellules à remanier. En ce qui concerne les cellules royales, les "façons de base » pour continuer à employer un langage voisin de celui de DÉLEURANCE, ne s'effectuent qu'en l'absence de larves. Et dans le cas d'orphelinage brusque, lorsque les ouvrières transforment en cellules royales une cellule d'ouvrière où se trouve déjà une larve, sa base n'est jamais remaniée. D'où il suit que la présence de jeune couvain est incompatible avec le remaniement de la zone de la cellule sur laquelle il repose; que les pulsions de remaniement l'emportent sur celles d'élevage et provoquent le rejet des larves.

Mais comment expliquer l'importance du bord libre qui n'apparaît qu'ici et non pas dans les cellules d'ouvrières, puisque les nourrices n'élèvent les larves d'ouvrières et les larves de mâles que dans des cellules à cloisons mitoyennes? Et d'autre part, pourquoi les ouvrières expulsentelles ou abandonnent-elles certaines larves même greffées dans des cellules d'ouvrières, en vue d'un élevage royal, au lieu de les élever tout au moins en ouvrières? Ce n'est point ici la position verticale avec ouverture dirigée vers le bas, qui peut les déterminer à se comporter de la sorte puisqu'on peut placer dans la position horizontale tous les rayons verticaux d'une ruche sans troubler le moins du monde l'élevage ou l'entrepôt des provisions, même au bout de plusieurs mois (CHAUvis, observation non publiée). Peut-être alors faut-il un certain degré d'accoutumance à la position verticale des cellules pour que les Abeilles acceptent d'y élever à la fois larves d'ouvrières et larves de reines (elles le font dans la ruche à rayons horizontaux).

\section{III. - LES CELLULES A DEUX LARVES}

Si la larve constitue un stimulus important, nous devons pouvoir en accroître 1'intensité : en plaçant non plus une, mais deux larves jeunes dans la moitié de nos cupules, et en les comparant à des cupules munies d'une seule larve dans l'autre moitié, nous devrions réduire les abandons dans le cas des cellules à deux larves. Mais pour les cellules à une larve, 
63 p. roo des cellules ont été terminées, avec une production de G. R. de Io,8 g pour roo larves greffées ( $37 \mathrm{p}$. roo de larves abandonnées). Avec deux larves, nous pensions obtenir $(37: 2)$ I 8 p. Ioo d'abandon en moins, soit 8I,5 p. Ioo de cellules acceptées. Mais en fait seulement $70 \mathrm{p}$. Ioo des cellules ont été acceptées, ce qui prouve que la présence de la larve ne détermine pas, à elle seule, les variations du pourcentage d'acceptation.

Dans les cellules à deux larves, nous avons récolté i I, $25 \mathrm{~g}$ de G. R. au lieu de Io,8; apparemment, la quantité de G. R. n'est donc pas modifiée : il est logique toutefois de penser que la secrétion a été en fait plus importante dans les cellules à 2 larves; car les larves se sont nourries pendant trois jours et ont consommé deux fois plus de G. R. que dans le cas de larves isolées. Nous trouvons en effet $0,16 \mathrm{r} g$ de G. R. par cellule à 2 larves contre $0, \mathrm{I} 73$ par cellule à I larve, et nous ne pouvons admettre que les larves n'ont mangé que $0,012 \mathrm{~g}$ en trois jours, puisque leurs poids sont tout à fait normaux. Donc, elles se sont nourries comme d'habitude et forcément, les nourrices ont dû leur fournir deux fois plus de G. R. par cellule.

Il est à noter par ailleurs que les larves abandonnées au finisseur sont toujours celles qui, à la sortie du starter, sont le moins bien pourvues en G. R. Nous avons vérifié ce fait en introduisant directement dans les finisseurs des larves déposées dans des cupules déjà familiarisées et contenant plus ou moins de gelée; et il est bien vrai que celles qui en renferment davantage sont élevées dans de meilleures conditions.

Parmi les 70 p. Ioo de cellules à 2 larves terminées, I4 contenaient encore 2 larves, alors qu'une des deux larves avait été retirée dans $5^{6}$ cellules par les abeilles. On peut se demander pourquoi les nourrices évacuent telle larve plutôt que telle autre. Mais nous ne disposons pas encore d'expériences là-dessus.

\section{Groupement de larves d'âge différent.}

Au lieu d'introduire directement dans chaque cellule deux larves au départ, nous avons ajouté une jeune larve d'un jour dans II cellules royales dont les larves initiales étaient âgées de trois jours. Exceptionnellement, les Abeilles ont été laissées libres d'operculer cette série. 'l'outes l'ont été effectivement mais une seule semblait, d'après son opercule à double tête (photo $\mathrm{n}^{\circ} \mathrm{Io}$ ), contenir deux larves. Mais à la dissection de la cellule, l'espace intérieur était divisé en det1x loges par le cocon qu'avait tissé la larve la plus âgée, qui se trouvait dans une partie de la cellule arrondie et de forme normale. La logette voisine, déterminée par l'expansion latérale de la cellule royale, était ride, et toute trace de la jeune larve en était absente. Les abeilles, en construisant le "chapeau " de forme anormale, n'ont pas édifié de cloison entre les deux larves pas 
plus qu'elles n'ont été capables de les séparer à la base dans la zone critique (voir discussion plus haut). Mais nous ne savons si la plus jeune larve a été retirée tardivement par les abeilles ou mangée par la plus grosse.

La même expérience a été refaite en ajoutant dans les cellules des larves royales de trois jours, des larves royales $d u$ même âge. 7 cellules sur I I ont été operculées; l'une d'elle renfermait encore deux larves. Ainsi donc, le groupement peut-être conservé au moins jusqu'à l'operculation, mais c'est fort rave.

\section{IV. - L'AGE DES LARVES GREFFÉES. NOUVELLES DONNÉES}

Si les Abeilles élèvent spontanément des reines à partir de larves de " pré-reines " très grosses (VUILLAUME, I957), et si elles construisent des cellules royales autour de ces larves, elles ne le font jamais spontanément autour de grosses larves d'ouvrières. Il est toutefois possible, bien que difficile, d'obtenir des cellules royales artificielles avec des larves d'ouvrières de plus de trois jours ; on peut même aller jusqu'à 6 jours : les vieilles larves se transforment alors non plus en reines typiques, mais en intermédiaires entre ouvrières et reines (ZANDER et BECKER). En essayant nous même de faire accepter des larves de différents âges, nous avons obtenu les résultats suivants :

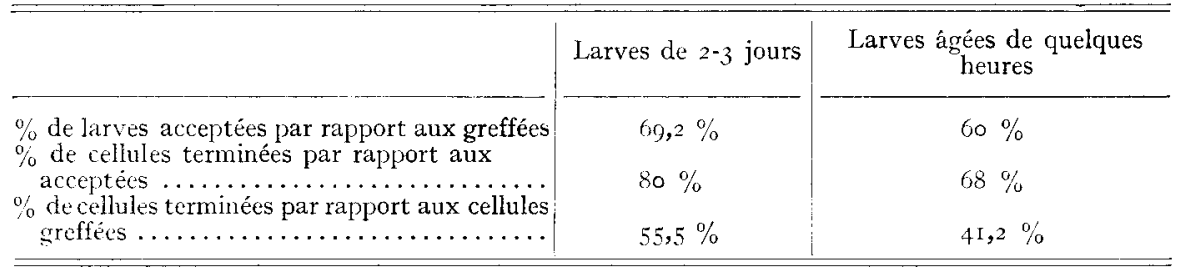

Nous obtenons donc, assez paradoxalement de meilleurs résultats avec des larves plus âgées. D'autre part, en introduisant directement dans les finisseurs sur une grande quantité de G. R. des larves très jeunes en compétition avec des larves de plus de trois jours, les nourrices élèvent indifféremment les unes et les autres. Rappelons que nous ne nous intéressons toujours ici qu'au stade initial de l'élevage, et non au stade final.

Nous avons vu aussi que les abeilles des finisseurs élèvent les larves qui sont passées au starter aussi bien que celles qui n'y sont pas passées (à condition d'augmenter la quantité initiale de G. R. déposée dans la cellule). Mais, lorsqu'on remplace la moitié des larves des cupules acceptées dans les starters par des larves "neuves" les pourcentages d'acceptation sont pourtant légèrement différents en faveur des larves les plus grosses (50,6 p. Ioo contre 42 p. Ioo). Par contre, des larves acceptées au starter, 
donc âgées de $\mathrm{I}$ à 2 jours, déplacées et mises dans des cellules refusées (ne contenant par conséquent ni larves ni $(\dot{i}$. R.) sont abandonnées.

Nous avons aussi laissé dans un starter une série de I2 grosses larves d'ouvrières que nous avions greffées dans des cellules royales juste avant leur operculation. Trois jours après, une seule larve subsistait, dans une cellule royale operculée. Les larves pourvues d'une plus grande abondance de réserves internes peuvent vivre plus longtemps sans soin et attendre peut-être que la quantité de substance d'acceptation atteigne le seuil favorable à l'élevage royal. Cette hypothèse peut se vérifier à notre sens en greffant des grosses larves royales ( 3 jours) sur de la G. R. pure ou sur un mélange dilué d'eau + G. R. Sur G. R. pure dans le starter, 25 p. Ioo des larves sont élevées tandis que toutes celles placées en compétition dans la même ruche sur eau + (.) R. sont abandonnées. I,e finisseur termine 62 p. Ioo de ces cellules acceptées.

Ein faisant la même expérience avec introduction directe des cellules dans un finisseur les mêmes proportions de cellules sont terminées.

\section{Larves de mâles.}

Rappelons que des larves de mâles logées dans des ébauches de cellules royales sont effectivement élevées (Vrillaume 1957). Placies en compétition dans une ruche, en présence de jeunes larves d'ouvrières de même âge, elles donnent les résultats suivants :

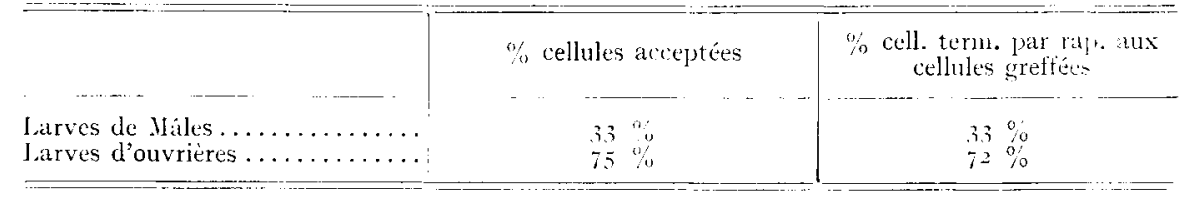

Nous avons déjà signalé que les cellules royales où l'on a fait élever des mâles affectent au moment de la nymphose une structure particulière (Vuiliauni 1957). I a nymphe de mâle se trouve alors logée dans une loge nymphale cylindrique ajoutée à la cellule royale nornale. Il existe entre les deux parties un étranglement, seul signe que les Abeilles reconnaissent la larve de mâle, mais seulement après trois jours de vie larvaire. Alors pour corriger la structure de la cellule royale oì se trouve un mâle elles ne démolissent rien, mais ajoutent une loge de màle ronde à la cellule royale normale, ce qui double presque la longueur de celle-ci.

Nous n'avons pas encore étudiée le comportement des Abeilles clevant des ébauches de cellules qui contiennent des œufs. Réagissent-elles de la même manière qu'en présence des larves? Nous ne pouvons l'affitmer encore. 


\section{V. - L'INFLUENCE DE LA REINE}

Nous avons signalé par ailleurs qu'une acceptation des cupules était possible dans une ruche pourvue d'une reine en pleine liberté, même en dehors de la période d'essaimage (VUILLAUME, I957). Rappelons à ce sujet que, suivant la théorie hormonale de BUTLER, l'ectohormone de la reine, léchée par les abeilles inhibe l'élevage royal dans tout le corps social. Les Abeilles n'élèvent naturellement des reines que dans des conditions bien définies :

- reine devenue trop âgée (bourdonneuse) à laquelle est alors substituée une jeune reine,

- colonie devenue trop populeuse et préparant un essaimage,

- ruche orphelinée accidentellement et brutalement,

Au cours de ces périodes critiques la source d'ectohormone se trouve tarie complètement ou partiellement ; un déséquilibre hormonal s'ensuit à l'intérieur de la ruche, et les abeilles édifient alors des ébauches de cellules royales. I a construction de ces ébauches et de ces ébauches seules, qui ne contiennent pas forcément d'œuf ou de larve au départ, est arrêtée normalement par l'ectohormone. Ce n'est qu'au moment de l'éclosion de l'œuf que commence le dégorgement de gelée royale et que la cellule s'allonge.

Or, dans une ruche finisseuse dont la reine est simplement éloignée des cellules royales à élever, par une grille à reine, l'ectohormone peut diffuser à travers la grille; effectivement, nous ne trouvons jamais de cellules royales naturelles dans la partie orpheline. Mais les abeilles continuent pourtant à édifier des " chapeaux " sur les cupules, garnies de larves, que nous leur présentons. Car, nous l'avons déjà dit, seul le début et non la fin de la construction des cellules royales est vraiment sensible à l'ectohormone.

Mais les Abeilles continuent aussi l'élevage des cupules dans une ruche ordinaire où la reine se trouve emprisonnée dans une cagette d'introduction reconverte de toile métallique. Ici non plus la reine n'inhibe pas la construction du chapeau ni le dégorgement de la G. R. Et le pourcentage de cellules terminées est important. Il est vrai que BUTLER est le premier à signaler que toute restriction apportée à la liberté d'accès desabeilles vers la reine restreint l'action de l'ectohormone.

I, 'ectohormone existe aussi dans les starters pourvus de jeunes reines fécondées; et aussi dans le cas des cellules introduites directement dans le nid à couvain d'une ruche normale. Toutefois, dans ce dernier cas, si les pourcentages d'acceptation sont très élevés lors de la première introduction, ils diminuent rapidement et de $80 \mathrm{p}$. Ioo environ, ils passent à Io p. Ioo au cours de la seconde opération, pour tomber aux environs 
de o la troisième fois. Assistons-nous à un rétablissement d'équilibre, à une régulation interne par une sécrétion plus intense de l'ectohormone royale? Ou, plus simplement la provision disponible de gelée des ouvrières est-elle réduite à cause de l'élevage normal déjà entrepris par ailleurs? On peut se demander alors ce qui arriverait si après la seconde introduction on enlevait tout le couvain non operculé. Il est vrai que dans un finisseur les Abeilles dégorgent de la gelée dans les cellules royales tout en élevant un couvain assez important, mais la population d'un finisseur est habituellement beaucoup plus forte que celle d'une ruche normale et la provision globale de gelée doit donc être plus considérable. De toute façon, le finisseur nous montre, comme nous l'avons vu, que la seule présence de la reine ne suffit pas à inhiber les acceptations.

Quant à la jeune reine qui peut se développer dans les starters, soulvent à l'insu de l'expérimentateur, elle doit être incapable au début de sécréter assez d'ectohormone, car nous avons pu faire démarrer des élevages royaux en sa présence avec des acceptations de plus de 50 p. roo sans diminution de ce pourcentage pendant I $_{5}$ jours consécutifs aussi bien en août qu'en septembre, donc pas forcément en période d'essaimage.

\section{La finition de la cellule royale est-elle tout à fait insensible à l'ectohormone?}

Nous avons dit plus haut que seule ou à peu près la phase initiale de la construction des cellules royales était inhibée par l'ectohormone. Toutefois, si on laisse, jusqu'à la finition des cellules déjà acceptées à l'intérieur du starter (dépourvu de reine, rappelons-le) les pourcentages de cellules terminées sont nettement supérieurs à ceux obtenus après transfert dans le finisseur (66 p. Ioo des cellules sont acceptées dans le starter et 50,I p. Ioo terminées contre 29,9 p. Ioo terminées dans le finisseur pendant la même période). La reine doit donc exercer tout de même une certaine inhibition dans le finisseur; mais cela n'est pas encore tout à fait sûr, car la simple influence du transfert dans une autre colonie pourrait provoquer peut-être une perturbation du même ordre; et des transferts de starter à starter seraient nécessaires avant d'accepter l'une ou l'autre hypothèse.

Notons aussi que si nous avons pu faire accepter des cellules royales dans des starters pourvus de jeunes reines fécondées nous n'avons jamais laissé ces cellules dans les starters jusqu'à l'éclosion ; et nous ignorons par conséquent si les abeilles les auraient tolé '́es jusqu'à la fin.

La gelée royale dégorgée dans les cellules que l'on laisse dans le starter est bien moins abondante que dans le finisseur, mais comme beaucoup plus de cellules sont acceptées, on récolte finalement des quantités analogues. 


\section{VI. - Élevage de Cellules Royales en PRÉSence DE VIEILLES CELLULES}

Si on laisse dans un finisseur des cellules royales vieilles de 3 jours et davantage, les abeilles peuvent entreprendre néanmoins à leur côté l'élevage de nouvelles cuptrles. Les pourcentages d'acceptations des nouvelles cellules ne sont nullement affectés, même en présence de plus de Ioo cellules déjà operculées. Si les premières cellules ne sont pas operculées les nourrices continuent à s'en occuper, tout en commençant l'élevage des jeunes larves nouvellement introduites. Elles entreprennent même cet élevage en présence de reines nouvellement écloses, pendant que commence juste à côté la destruction des cellules contenant des reines prêtes à éclore (photo 12). Les stimuli qui amènent les Abeilles à détruire des cellules royales âgées peuvent donc coexister dans une ruche avec les stimuli commandant les élevages des nowvelles cellules. Ne sont détruites que les vieilles cellules royales âgées de I4 jours (y compris le stade œuf). C'est donc juste avant la naissance de la jeune reine que la cellule peut être attaquée. Il n'est pas impossible que les bruits émis alors par l'insecte emprisonné dans sa cellule jouent un certain rôle dans le déclenchement des attaques. Signalons que dans ces expériences les cellules âgées ou en voie de destruction étaient portées par le même cadre porte-cellules que les jeunes (et donc à proximité immédiate). De plus, des cupules faites avec la cire des cellules royales dont les reines sont déjà écloses en voie de destruction sont moins bien acceptées que les cupules normales: phénomène intéressant que nous nous réservons d'étudier plus à fond.

\section{VII. - ROLE DE LA SUBSTANCE D' IN HIBITION ET D'ACCEPTATION DANS L'ÉLEVAGE ROYAL}

I'ectohormone secrétée par la reine, déposée en solution alcoolique sur des cellules de verre dans lesquelles nous introduisons ensuite des larves, inhibe fortement l'élevage royal.

\begin{tabular}{|c|c|c|}
\hline & + substance inhibitrice & Sans substance \\
\hline Greffées $\ldots \ldots \ldots \ldots \ldots \ldots \ldots$ & 100 & 100 \\
\hline 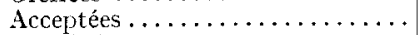 & 4 & 22 \\
\hline Terminées................... & 2 & I 2 \\
\hline
\end{tabular}

Des résultats aussi nets sont obtenus en remplaçant la solution alcoolique d'ectohormone par une solution acétonique de propolis. Son pouvoir inhibiteur est très grand. Nous avons tenté d'en extraire le ou les principes 
actifs, mais auparavant, nous avons pensé que les mauvais résultats donnés par certaines cires d'abeilles au moment de l'acceptation étaient peutêtre dus à des traces de propolis dans la cire.

Des cellules neuves en cire, non familiarisées, renfermant tous les composés acétono-solubles, (cire extraite des rayons par l'acétone) mises en compétition avec des cupules dépourvues de composés acétono-solubles (cire extraite des rayons par l'eau chaude), donnent les résultats suivants :

\begin{tabular}{|c|c|c|}
\hline & Sans les composés solubles & $\begin{array}{c}\text { Avec les composés } \\
\text { solubles }\end{array}$ \\
\hline 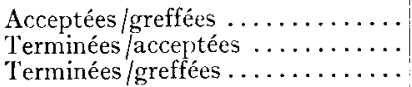 & $\begin{array}{l}65,6 \% \\
10 \\
6\end{array}$ & $\begin{array}{c}25 \% \\
0 \\
0\end{array}$ \\
\hline
\end{tabular}

Nous avions employé dans cette expérience des cellules non familiarisées, ce qui explique les faibles résultats obtenus même dans le cas des cupules sans composés acétonosolubles; on voit néanmoins sur le tableau la forte inhibition qu'exercent ces composés. Sans les dérivés en question, 62 p. Ioo des cellules greffées sont acceptées, 40 p. Ioo des cellules acceptées sont terminées, et $25 \mathrm{p}$. Ioo des greffés sont terminées.

Mises en compétition avec les cellules familiarisées, les cellules de cire avec dérivés acétono-solubles donnent des résultats plus nets encore:

\begin{tabular}{|c|c|c|c|}
\hline & $\% A / G r$ & $\% \mathrm{~T} / \mathrm{Gr}$ & $\% \mathrm{~T} / \mathrm{A}$ \\
\hline 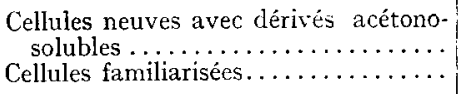 & $\begin{array}{c}6 \\
5^{6,2}\end{array}$ & $\begin{array}{r}0 \\
28\end{array}$ & $\begin{array}{r}0 \\
50\end{array}$ \\
\hline
\end{tabular}

Il est donc bien évident que la substance d'inhibition passe dans les cires extraites des rayons par l'acétone.

\section{Substance d'acceptation (Vuillaume, 195\%), (Photo $\mathrm{n}^{\circ} \mathbf{1 1}$ ).}

Comme dans le cas de la propolis, nous testons la substance d'acceptation sur des cupules neuves de verre. Des cellules familiarisées sont terminées à 78 p. roo alors que les non-familiarisées le sont à $I_{5}, 6$ p. roo seulement. Nous avons alors essayé d'extraire la substance de familiarisation et de l'ajouter sur des cupules neuves.

D'abord, nous avons vérifié que des cupules de verre ou de cire récupérées (c'est-à-dire utilisées une deuxième fois et donc enduites en principe de substance d'acceptation) et mises en compétition sont acceptées par les Abeilles dans la même proportion. Mais des larves déposées dans des cupules de verre neuves ou récupérées sont acceptées dans les mêmes proportions. Ceci montre qu'en l'absence de substance d'inhibition, (les cellules de 
verres neuves n'en ont pas évidemment) la substance d'acceptation n'est pas indispensable; dans les cupules de cire sa présence vient seulement contrarier l'action de la substance d'inhibition qui imprègne plus ou moins toutes les cires d'abeilles (mais évidemment pas le verre des cupules neuves.)

Un lavage à l'eau des cellules de verre récupérées n'entraîne pas la substance d'acceptation; mais l'alcool l'entraine plus ou moins ainsi que 1'acétone: dans ce dernier cas, 30 p. roo de cellules lavées introduites directement dans le finisseur sont terminées contre $57 \mathrm{p}$. roo de cellules non lavées. La quantité de gelée récoltée dans les cellules lavées est d'environ le quart de celle récoltée dans les cellules non lavées. Avec la méthode directe (introduction dans le. finisseur sans passer par le starter) les résultats sont beaucoup plus nets encore avec 1'alcool : toutes les cellules lavées à l'alcool sont abandonnées et 33 p. Ioo des cellules témoins élevées. L'alcool serait donc un meilleur solvant de la substance que l'acétone. Un lavage au benzène n'a pas d'effet.

Mais en essayant d'ajouter à des cupules neuves cette substance d'acceptation, nous n'avons pas obtenu de bons résultats, ni de différences significatives avec les cupules lavées, alors que les badigeonnages avec la substance d'inhibition réussissent très bien :

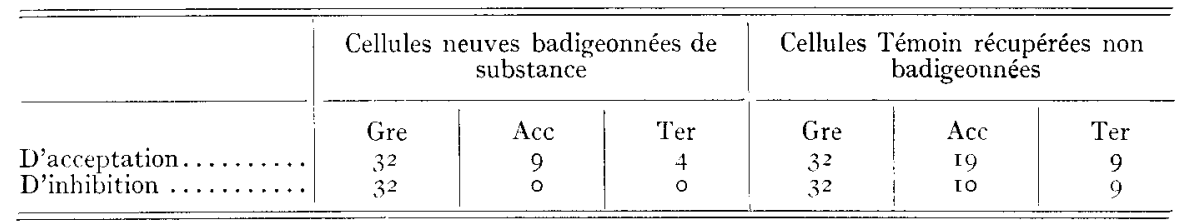

\section{Cellules refusées et reformées.}

La structure des cellules refusées est toujours légèrement modifiée par les Abeilles au cours de leur séjour dans le starter et finisseur (3 jours). Pendant ce temps, les Abeilles déposent certainement de la subtance d'acceptation, mais elles ferment partiellement l'ouverture de la cellule. Ce sont ces cellules que nous avons, après avoir élargi leur ouverture, comparées avec les cellules récupérées.

\begin{tabular}{|c|c|c|}
\hline & Cellules terminées & Cellules refusées et reformées \\
\hline $\begin{array}{l}\text { Acceptées/greffées ........ } \\
\text { Terminées/acceptées ....... } \\
\text { Terminées/greffées. . . . . }\end{array}$ & $\begin{array}{l}67,9 \% \\
66,6 \% \\
45,2 \%\end{array}$ & $\begin{array}{l}44,5 \% \\
50,8 \% \\
22,6 \%\end{array}$ \\
\hline
\end{tabular}

Ceci montre un avantage certain en faveur des cellules non refusées. Est-ce dî̀ à des différences de quantité de substance d'acceptation ou bien au rôle de la gelée royale dont nous avons déjà parlé? En effet, les cellules 
récupérées ont contenu une larve et de la gelée royale. En prélevant l'une et l'autre, nous laissons, malgré tous les soins qu'on peut apporter, une certaine quantité de gelée royale qui s'ajoute au mélange introduitau moment du“ picking ", alors qu'avec les cupules refusées, la larve est déposée sur la même quantité de mélange que celle mise dans les cellules acceptées.

\section{Présence du cocon à l'intérieur des cellules royales.}

Nous a vons utilisé également des cellules royales d'où la reine est sortie: cellules contenant par conséquent un cocon. Naturellement, les abeilles ne se trouvent jamais devant un tel cas, puisqu'elles détruisent la cellule dès qu'une reine est née. Nous avons extirpé ce cocon à l'intérieur de cellules royales et les avons mises en compétition avec des cellules royales ayant gardé leur cocon et des cellules récupérées, cellules qui n'ont par conséquent jamais eu de cocon. Les résultats obtenus sont figurés dans le tableau suivant :

\begin{tabular}{|c|c|c|c|}
\hline & Avec cocon & Cocon enlevé & Récupérées \\
\hline $\begin{array}{l}\text { Acceptées/greffées ........ } \\
\text { Terminées/acceptées ....... } \\
\text { Terminées/greffées....... }\end{array}$ & $\begin{array}{l}87 \% \\
21,5 \% \\
18,7 \%\end{array}$ & $\begin{array}{l}88 \% \\
60 \% \\
5.3 \%\end{array}$ & $\begin{array}{l}42 \% \\
76 \% \\
33 \%\end{array}$ \\
\hline
\end{tabular}

Les acceptations sont nettement favorisées chez les cellules royales qui ont été terminées. Elles possèdent peut-être davantage de substance d'acceptation que les cellules récupérées.

La présence du cocon intervient surtout dans le finisseur.Avec cocon, 21,5 p. Ioo des cellules acceptées sont terminées tandis que $60 \mathrm{p}$. Ioo le sont si nous enlevons le cocon. Dans la même ruche, $76 \mathrm{p}$. Ioo des cellules acceptées récupérées sont terminées au même moment. Le cocon joue donc un rôle néfaste dans les élevages royaux.

Ceci est très différent de l'élevage des ouvrières où toutes les cellules sont tapissées intérieurement par une quantité toujours plus importante de cocons puisqu'au fur et à mesure que les Abeilles adultes éclosent, la reine vient pondre à nouveau dans les mêmes cellules.

\section{CONCLUSION}

Tout en éclaircissant certains faits (importance de la gelée royale, de 1'âge de la larve, de la disposition des cupules). nous voulons dans ce deuxième travail poser quelques problèmes importants que nous espérons pouvoir résoudre au cours des prochaines saisons apicoles. Des observations récentes faites par I ECOMTE montrant l'existence à l'intérieur de la ruche de toute une série de substances propres à une colonie ou propres 
à toutes les colonies vont nous aider, espérons-le. I,e rôle de substances d'inhibition et d'acceptation, prouvé d'une façon suffisamment nette, nous laisse entrevoir un programme de recherches assez chargé. I.e fractionnement de l'ecto hormone entrepris par J. Parn de même que les fractionnements que nous avons ébauchés, de la propolis, fractionnements que nous accompagnons de l'étude de différentes substances végétales (résines de bourgeons ou d'écorce) (voir note Vuil.AUME) ainsi que les résultats obtenus sur la construction cirière en général (V'cilis.dune et DARCHEN) devraient nous aider également dans la compréhension de ces phénomènes si complexes.

\section{RESUMÉ}

Io I a présence de gelée royale pure ou à forte concentration dans les cupules artificielles favorise l'acceptation des jeunes larves, mais surtout augmente le pourcentage des finitions, ainsi que la quantité de G. R. dégorgée ultérieurement par les nourrices. Le greffage sur G. R. pure permet l'introduction directe dans le finisseur sans passage par le starter, avec un très bon pourcentage de réussites.

$2^{\circ}$ La substitution de larves très jeunes à des larves déjà âgées de trois jours dans les cellules royales amène un nouveau et très important dégorgement de G. R. sur celle qui existait déjà. Une troisième substitution n'amène pas de nouvel apport de G. R., mais les nouvelles larves sont encore très bien élevées.

$3^{\circ}$ L'élevage échoue totalement lorsqu'on présente aux abeilles de très grandes cupules arrondies ou allongées, d'une taille excédant de beaucoup la normale, et dans lesquelles on introduit plusieurs larves à la fois, côte à côte ou séparées par des cloisons. La cause de l'inhibition pourrait résider en partie dans la forme anormale du fond (rayon trop grand) ; les abeilles y sont très sensibles et ne semblent pas pouvoir la modifier. Dans le cas des grandes cupules cloisonnées, un autre facteur inhibiteur se rencontre dans le fait que les cloisons ont forcément des parois mitoyennes; or, le bord des cellules royales doit être libre. I es nourrices tolèrent le rapprochement des bords, mais non pasleur fusion, comme nous le montrons à l'aide de diverses expériences.

$4^{\circ}$ Dans le cas des cellules d'ouvrières, les Abeilles peuvent faire subir au bord de profondes modifications, ainsi qu'à toute la cellule, mais ceci uniquement avant la ponte, lorsque les cellules sont vides; la présence de jeunes larves empêche toutes les modifications, qui ne reprennent qu'après leur expulsion.

$5^{\circ}$ I a greffe de deux larves à la fois dans les cupules normales n'augmente pas le pourcentage d'acceptation, mais incite les abeilles à sécréter 
davantage de gelée royale; mais à l'operculation une seule des deux larves subsiste.

$6^{\circ}$ L'âge ou la taille des larves greffées ne paraît pas d'une importance aussi grande qu'on le croit communément; et il est possible de faire accepter des larves fort grosses, à condition de les greffer sur G. R. pure ou très concentrée.

$7^{\circ}$ Seule la phase initiale de la construction spontanée des cellules royales est très sensible à l'ectohormone royale inhibitrice. Mais celle-ci n'agit guère sur l'acceptation ou la finition des cupules artificielles. Elles sont élevées dans la partie sans reine du finisseur, dans une ruche dont la reine est emprisonnée dans une petite cagette, et même en présence d'une reine âgée en pleine période de ponte: mais dans ce dernier cas, seule la première série de cupules est acceptée, et l'on n'obtient plus rien avec une seconde série.

$8^{\circ}$ Les cupules à finir peuvent être introđuites à côté d'autres cupules en cours d'operculation; ou même à côté de cellules mûres en voie de destruction parce que la jeune reine vient d'éclore ; l'élevage se poursuit néanmoins d'une façon normale, si bien que les stimuli qui poussent les abeilles à élever des cellules royales et ceux qui les déterminent à les détruire peuvent jouer côte à côte dans la ruche.

$9^{\circ}$ Le facteur inhibiteur que nous avions déjà signalé dans les cires extraites de rayons par l'acétone se confond avec la propolis; et plus spécialement avec une essence entraînable par la vapeur d'eau, qu'on peut extraire de cette substance après saponification.

IO ${ }^{\circ} \mathrm{I}_{1} \mathrm{a}$ substance d'acceptation déjà signalée également, paraît entrainable par l'acétone et non par le benzène.

I I $^{\circ}$ La greffe de nouvelles larves dans les cellules qui ont été refusées ne donne qu'un pourcentage médiocre d'acceptations. I a présence du cocon de la reine dans une cupule ne gêne guère l'acceptation, mais beaucoup la finition. 


\section{SUMMARY}

I $^{\mathrm{O}}$ Presence of pure or highly concentrated royal jelly in artificial cells favours the acceptation of young larvae, and increases the percentage of finished cells, so as the quantity of royal jelly further disgorged into. The introduction of one larva on pure royal jelly permits the direct introduction into the finisher, without previous passage into the starter, with a very high acceptation percentage.

$2^{\circ}$ Substitution of young, three days old larvae, in royal cells brings on a new and very important disgorgement of royal jelly upon the ancient jelly. A third substitution is without effect on this disgorgement, but larvae are bred very well.

$3^{\circ}$ We obtain no breeding when we present several larvae in broad and round wax cups or long cups $(2 \mathrm{~cm}$ diameter, or $9 \mathrm{~mm}$ diameter and 6 or $8 \mathrm{~cm}$ length) with or without partition between larvae. The inhibition factor is perhaps the unusual form (very important for the bees) of the bottom of the cells : bees are unable to modify it, especially without initial partition between larvae. With partition, especially in long cups, the partition is boundary. It is here an other important factor because royal cells edges must be free and without contact with the neighbours. 'The breeding is induced in royal cells if their edges are very close but not confounded.

$4^{\circ}$ In case of workers cells, bees are able to modify cells edge, but only before egg-laying, when the cells are empty. Presence of young larvae inhibits every modifications which begin again after their expulsion.

$5^{\circ}$ The introduction of 2 larvae in one ordinary cell does not increase the percentage of acceptation, but incites the bees to a more important production of royal jelly ; but at the closing of the cell, one larvae only remains.

$6^{\circ}$ The age of grafted larvae is not of great importance, and bees accept o to 6 days old larvae. In order to be accepted, the older must be put on pure royal jelly.

$7^{\circ}$ The initial phase only of the spontaneous building of royal cells is very sensible to the queen substance. Its action is weak on acceptation and finition of artificial cells, which are placed in the queenless part of a hive, or in a hive which queen is imprisoned in a little cage. The same phenomene is observed if the queen is free and young (less ${ }_{5} 5$ days) or with a normal queen during the period of swarming or a great laying. During this phase, the first serie only is always well accepted.

80 'The accepted cells can be introduced near other older cells, closed or not and even in presence of new-born queens, when bees and young queen are busy to destroy the older cells. So the stimuli for construction and destruction are different and can function simultaneously in the hive.

$9^{\circ}$ The inhibitor factor that we found in the wax (aceton-extraction) seems to blend with propolis.

Io 'The acceptation substance is soluble in aceton and not in benzen.

I I $^{\circ}$ The introduction of new larvae in cells which were not accepted gives a low percentage of acceptation.

Presence of cocoon of queen in the cells does not inhibit the acceptation of larvae, but troubles the finition. 


\section{RÉFÉRENCES BIBLIOGRAPHIQUES}

Darchen (R.) et Vuiliaume (M.). - Les pré-reines chez Apis mellifica. Communication Congrès International de 1'U. I. E. I.S. Juillet I957 Paris, I957.

DaRchex (R.) Vizier (Cl.) et Vuillaume: (M.). - Sur le déterminisme de la construction des cellules de mâles chez les Abeilles. C. R. Ac. Sc., 14, I, P. 39I-394.

DELEURANCE (E. P.). - Contribution à l'étude du comportement des Polistes. Thèse Paris, I957.

Gorilot (Ch.). - Étude physique de la conservation de la gelée royale brute. C.R. Ac. Sc., T, 245, P. Io82-84, I957.

Vuiriadue (M.). - Contribution à la psychophysiologie de l'élevage des reines chez les Abeilles. Insectes Socianx, VI, ${ }^{\circ}$ 2, I957.

VuILLACME (M.). - La forme des cellules royales chez les Abeilles, id, no 4, 1957 ZANDER (E.) et BECKER (F.). - Die Ausbildung des Geschlechtes bei der Honigbiene. Erlanger Jahrbuch fïr Bienenkunde., Band 3, P. PareyBerlin, I925. 


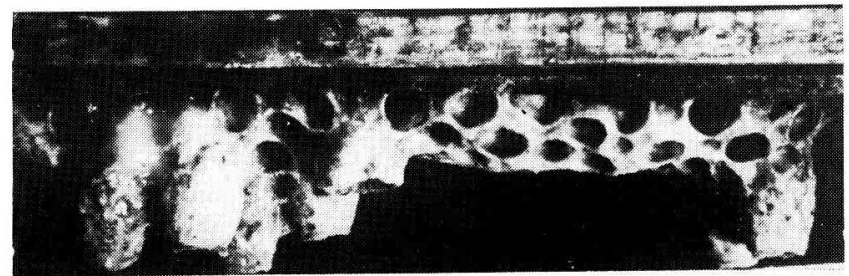

pното т. - Cupules normales placées côte à côte sur 3 rangées (de profil).

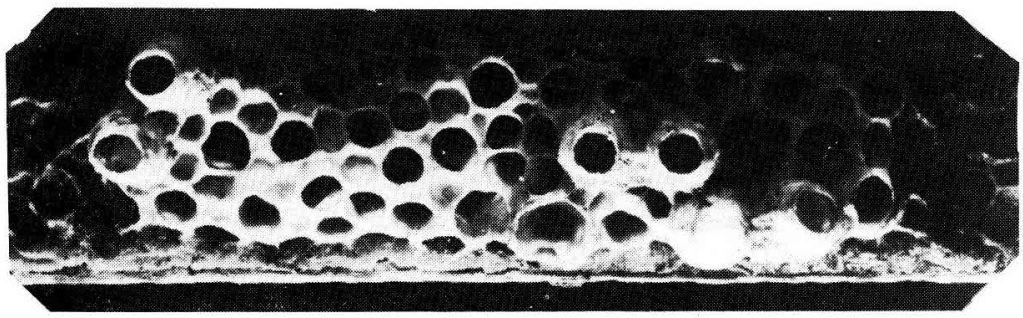

I’IOTO 2. - Cupules nommales placécs cote à côte sur 3 mangies (vue de dessus). Noter la présence du bord libre entre les cellules rovales étirćes ainsi que l'ouverture ronde des collules d'ouvrières ( 5 mm de diamitre) construites entre les celiules royales.

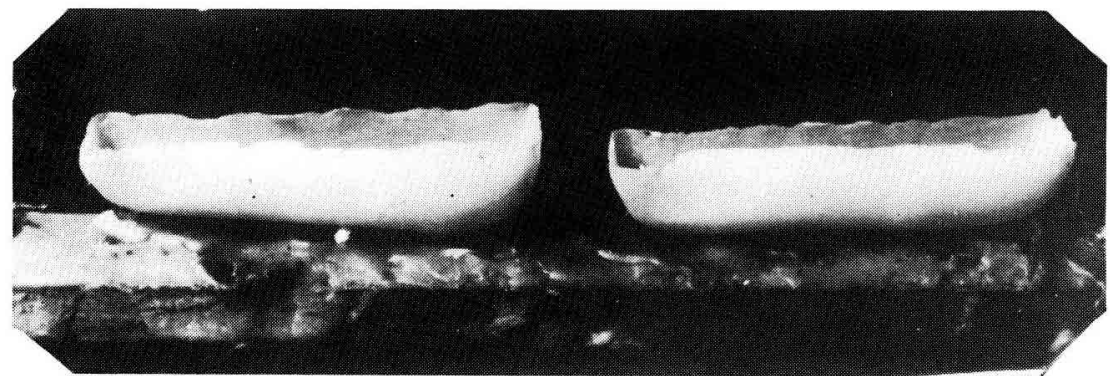

Pinoto 3. - Barquettes saus cloisons avant leur introduction dans une ruche. (de profil).

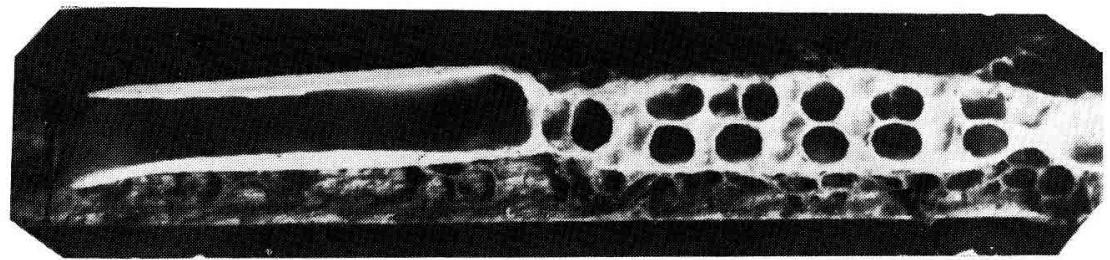

Pнoто 4. - Barquetes dont l'une avec cloison modifiée. Noter conme sur la photo no 2 la forme ronde de l'ouverture des cellules d'ouvrières construites par les abeilles à la place des barquettes cloisonnées. Nous n'avions placé ici que des cloisons transversales, délinitant 6 cellules à section ¿̀ peu près carrée. A droite barquette sans cloisons initiales non encore modifiée. 


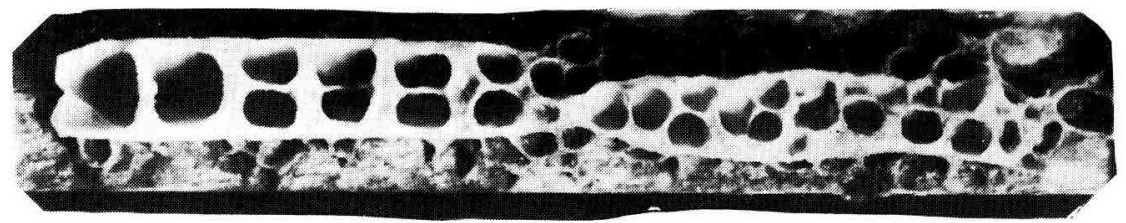

P'ro'vo 5.-.- Barquettes modiliées par les abeilles, I'une sans cloisons initiales (á droite). Noter l'irrégularité des cellules rondes. (ne autre aver cloisons initiales (at galuhe). Les modifications sont moins avancées que sur l'inawe corresponclante de lit photo 4 . On apercoit dans la 2 e calluke ì jartir de la gauche, une ébauche de cloison construite par les abeilles, et qui, plus tard, diviserat la collule initiale en 2 cellules d'ouvrieres.

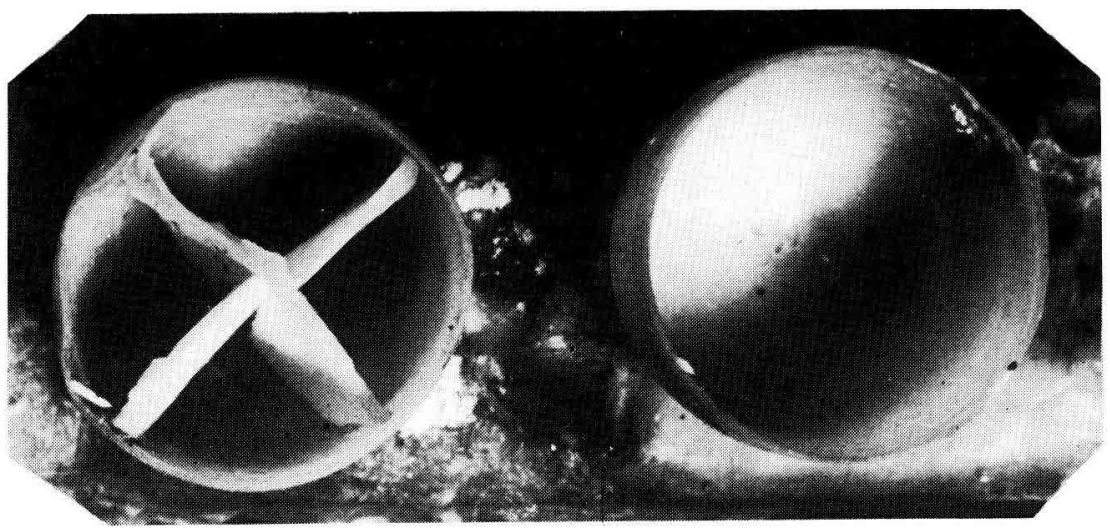

Phoro 6. -... Grosses cupules avec et sans robons avant leur introduction dans la nuche.

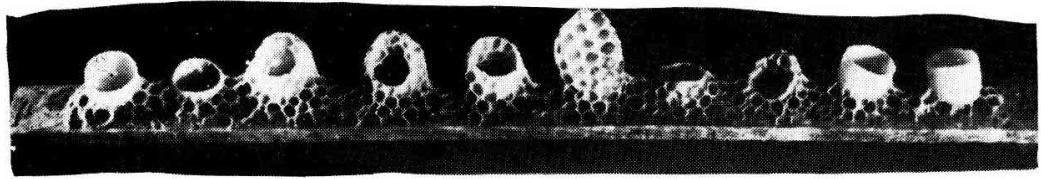

Pioto 7. - Grosses cupules sans doisons, apris passiane dans me ruche. Noter les manchons de cellules d'ouvrieres que les Abeilles construisent autour de ces cupules. 


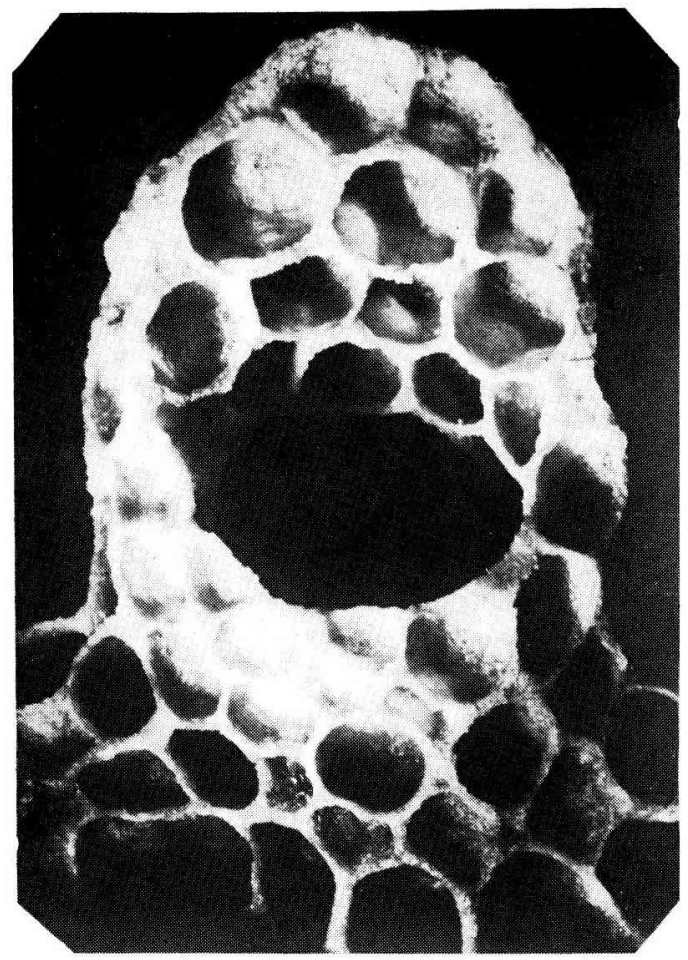

1.37

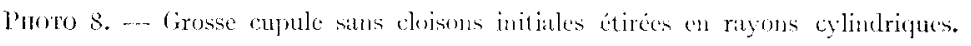

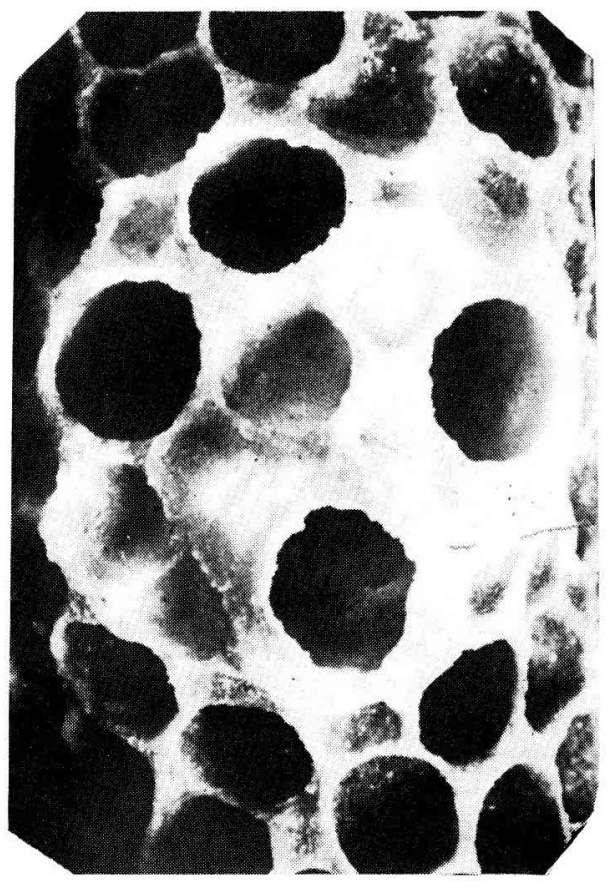

Pluto 9. Grosse cupule divisée en 4 par des cloisons inititilts ct modifiées par les abcilles en +1 cellules rondes. 


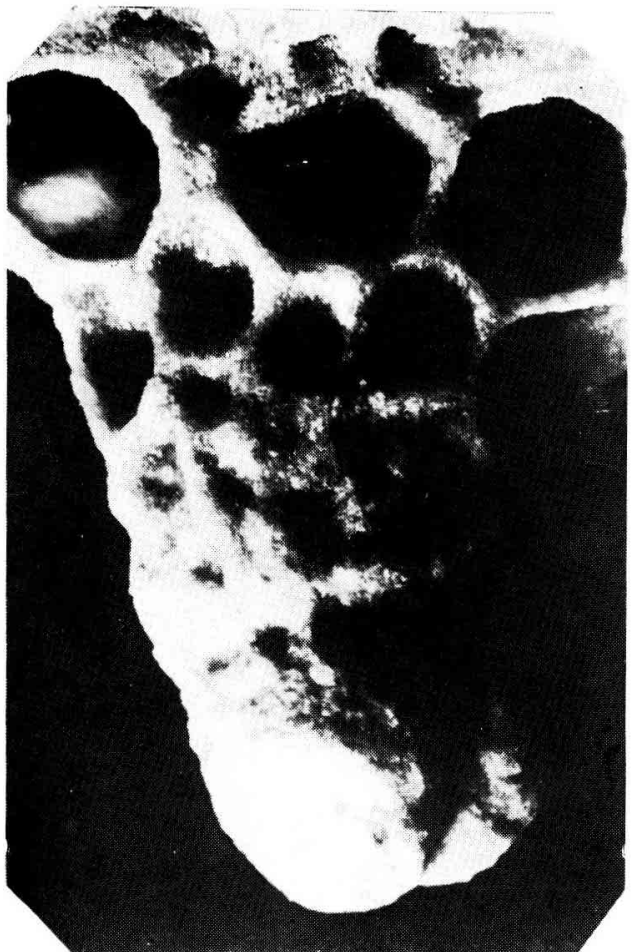

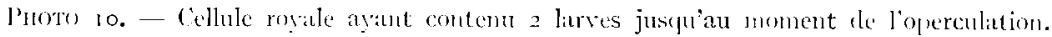

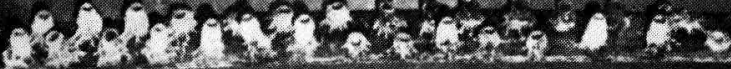

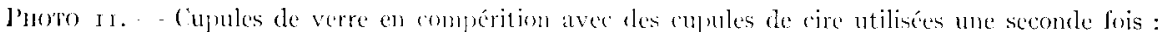

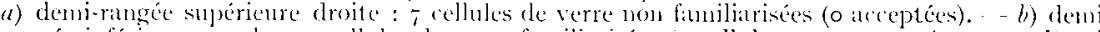
rangée inférieure gatuche : 7 cellules de verre fandiarisées (5 cellules sur 7 aceptées). - - e) Iteni

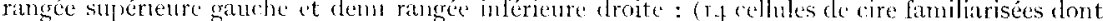
5 ont bé acerentes).

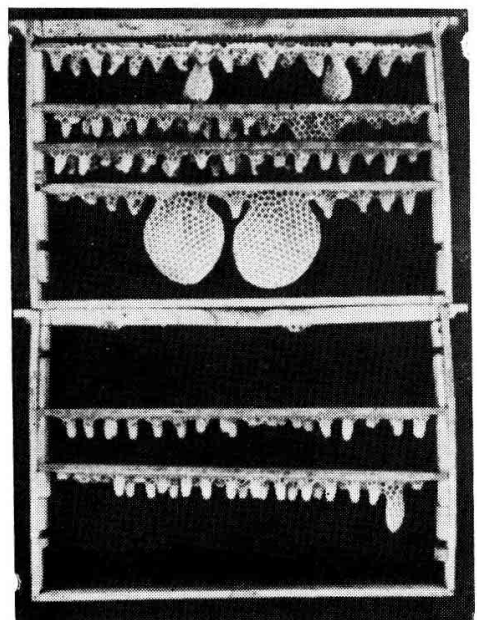

Puoro I2. - a cadres porte cellules introduits dans une ruche en mine temps: celui du haut mani de cellules royales dont les reines ćtaient prêtes à raître (Noter les cullules détruites). ('ului du has : larves royales de moins de trois jours qui ont été ćlevées alors que les cellules jlus âtries se faisaient détruire. 